\title{
July 2018 Critical Care Case of the Month
}

Stephanie Fountain, MD

Banner University Medical Center Phoenix

Phoenix, AZ USA

\section{History of Present IIIness}

A 45-year-old man was brought to the Emergency Room by his mother complaining of weakness, dizziness, and trouble swallowing. He was also incontinent of stool and looked "sunburned".

\section{Past Medical History}

He has a past medical history of:

- Schizophrenia

- Crohn's disease

- Depression

- Polysubstance abuse

- Type 2 diabetes

- Hyperlipidemia

\section{Medications}

- Prazosin

- Venlafaxine

- Risperidone

- Buspirone

- Oxcarbazepine

- Gabapentin

- Hydroxyzine

- Lithium

- $\mathrm{KCL}$

- Metformin

- Atorvastatin

- Adalimumab

- Mesalamine

- Prednisone

- Ferrous sulfate

\section{Physical Examination}

- Vitals: $80 \mathrm{~kg} / 97.3$ degrees / 101 bpm / 100\% 28RR / BP 111/72

- The patient was toxic appearing and flushed

- Oriented to self only, very lethargic

- Dry mucous membranes

- Lungs clear to auscultation and percussion

- Heart tachycardic but no murmurs

- Abdomen without organomegaly, masses or tenderness

- Extremities without edema

Which of the following should be done at this time?

1. Electrolytes

2. Lumbar puncture

3. Urine drug screen

4. 1 and 3

5. All of the above 


\section{Correct!}

\section{1 and 3}

The cause of the patient's change in mental status is unclear. A mnemonic for possible causes of altered mental state is AEIOU TIPS with each letter of the mnemonic standing for the following (1):

- A - Alcohol/Acidosis

- E -Electrolytes/Encephalopathy/Endocrine/Epilepsy/

- I - Infection- meningitis, encephalitis, sepsis, septic shock; pneumonia, urinary tract infection, occult osteomyelitis.

- O - Opiates, Overdose

- U-Uremia/Underdose

- T - Trauma - head injury, blood loss (shock).

- I - Insulin (hyper- or hypoglycemia)

- P-Poisoning/Psychosis/pharmacology

- S - Stroke/Seizure/syncope

Lumbar puncture may be indicated but is not indicated at this time because of lack of physical evidence of fever, meningismus, and radiography (CT scan, MRI) suggesting an indication. Furthermore, if the CT scan showed focal intracranial bleeding a lumbar puncture might result in herniation of the brainstem.

A complete blood count was performed and showed an elevated white blood cell count of WBC 19,000 cells/ $\mu \mathrm{L}$ ( $76 \%$ segs, $17 \%$ bands) but hemoglobin was normal (13.5 $\mathrm{g} / \mathrm{dL})$ and platelet count slightly elevated (506,000 platelets $/ \mu \mathrm{L})$.

A chest $\mathrm{x}$-ray was performed (Figure 1).

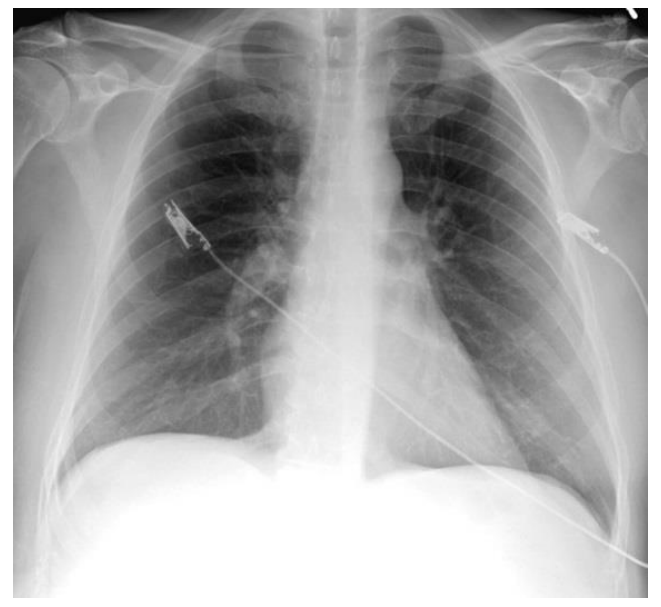

Figure 1. Initial portable AP of chest. 
A summary of the electrolytes, liver function tests, etc. with a comparison to values obtained 6 weeks earlier is shown in Table 1.

Table 1. Summary of laboratory values obtained in the emergency department (ED) and 6 weeks earlier during a routine outpatient visit (abnormal values in red).

\begin{tabular}{|c|c|c|}
\hline \multicolumn{2}{|c|}{ ED labs 10:30 am } & \multirow{2}{*}{$\begin{array}{c}6 \text { weeks prior } \\
84\end{array}$} \\
\hline GLUCOSE & 395 & \\
\hline BUN & 10 & 7 \\
\hline Creatinine & 1.43 & 0.71 \\
\hline SODIUM & 129 & 131 \\
\hline POTASSIUM & 4.7 & 4.1 \\
\hline CHLORIDE & 100 & 97 \\
\hline$\underline{\mathrm{tCO} 2}$ & 5 & 26 \\
\hline CALCIUM & 11.4 & 9.3 \\
\hline MAGNESIUM & 1.7 & 1.9 \\
\hline PROTEIN & 8.2 & 8.0 \\
\hline ALBUMIN & 4.0 & 3.3 \\
\hline ALKALINE PHOS & 166 & 250 \\
\hline AST & 10 & 20 \\
\hline $\mathrm{PO} 4$ & 4.9 & 3.9 \\
\hline$\underline{\text { ALT }}$ & 14 & 17 \\
\hline BILIRUBIN. TOTAL & 0.1 & 0.4 \\
\hline CPK & 22 & \\
\hline AMYLASE & 23 & \\
\hline LIPASE & 14 & \\
\hline
\end{tabular}

Which of the following acid-base disturbances are present?

1. Elevated anion gap metabolic acidosis

2. Elevated anion gap metabolic alkalosis

3. Low anion gap metabolic acidosis

4. Normal acid-base status

5. Normal anion gap metabolic acidosis 


\section{Correct! \\ 1. Elevated anion gap metabolic acidosis}

His tCO2, or bicarbonate, is low indicating a metabolic acidosis. Metabolic acidosis is separated into two categories-normal anion gap metabolic acidosis and elevated anion gap metabolic acidosis. The anion gap (AG) is calculated by subtracting the concentration of two anions, chloride $\left(\left[\mathrm{Cl}^{-}\right]\right)$and bicarbonate $\left(\left[\mathrm{HCO}^{-}\right]\right)$from the concentration of the major cations, sodium $\left(\left[\mathrm{Na}^{+}\right]\right)$and potassium $\left(\left[\mathrm{K}^{+}\right]\right)(2)$ :

$$
A G=\left(\left[\mathrm{Na}^{+}\right]+[\mathrm{K}+]\right)-\left(\left[\mathrm{Cl}^{-}\right]+\left[\mathrm{HCO}^{-}\right]\right)
$$

The anion gap is normally $<12 \mathrm{mEq} / \mathrm{L}$. Our patient's anion gap is elevated $\mathrm{AG}=(129+$ $5)-(100+5)=29$. A normal anion gap metabolic acidosis usually indicates a loss of $\mathrm{HCO}^{-}$most commonly through the gastrointestinal tract. An elevated anion gap indicates a metabolic acidosis caused by an acid, which has been titrated by the body's bicarbonate-carbonic acid buffer system and released its unmeasured anion.

Which of the following would be useful in determining the cause of the patient's elevated anion gap metabolic acidosis?

1. Serum lactate

2. Serum osmolal gap

3. Urine ketones

4. 1 and 3

5. All of the above 


\section{Correct! \\ 5. All of the above}

There are a number of common causes of an elevated anion gap metabolic acidosis which can be quickly excluded. Diabetic ketoacidosis is possible because the patient has diabetes and an elevated blood sugar, however, his urine ketones were negative. Lactic acidosis is commonly do to poor tissue perfusion and our patient has another potential cause, his metformin which can cause a lactic acidosis.

One way of narrowing the differential diagnosis is by calculating the osmolal gap. Sodium, chloride, bicarbonate, glucose, and urea are normally the most important osmotically active particles in the serum. The calculated serum osmolality is determined by (3):

Serum Osmolality $=(2 \times(\mathrm{Na}+\mathrm{K}))+(\mathrm{BUN} / 2.8)+($ glucose/18) + serum $($ alcohol/4.6)

A difference between the calculated osmolality and the measured osmolality of $>10$ mOSM indicates the presence of a molecule significantly raising the serum osmolality. Our patient's calculated serum osmolality calculated osmolality is $(2 \times(129+4.7))+(10$ / 2.8 $)+(395 / 18)+(0 / 4.6)=292$ mOSM but his measured osmolality was 333 mOSM.

Which of the following are the common cause(s) of an anion gap acidosis with an elevated osmolal gap?
1. Ethanol
2. Ethylene glycol
3. Methanol
4. 1 and 3
5. All of the above 


\section{Correct! \\ 5. All of the above}

There are a number of causes of a metabolic acidosis with an elevated anion and osmolal gap. These include, severe circulatory shock, hyperosmolar therapy, lactic acidosis, and chronic kidney disease, however, most of these are easily clinically recognizable (4). However, ingestion of the alcohols is a common clinical cause of metabolic acidosis with elevated anion and osmolal gaps that are not always easily diagnosed. Laboratory evaluation for the alcohols is often not readily available other than ethanol and therapy should begin prior to laboratory confirmation. Our patient's ethanol level was $0 \mathrm{mg} / \mathrm{dL}$.

Ethylene glycol and methanol result in a large osmolal gap while ethanol usually results in a small or no osmolal gap $(5,6)$. Fluorescein is usually added to antifreeze which is the most common cause of ethylene glycol ingestion to make detection of leaks in liquid cooled engines easily detectable. This can be taken advantage of by examining the patient's urine under a black light which can be available as a Wood's lamp. In addition, metabolites of ethylene glycol can accumulate and form calcium oxalate crystals in the urine.

Which of the following can be used to treat organic alcohol ingestion?

1. Hemodialysis

2. Inhibition of alcohol dehydrogenase

3. Sodium bicarbonate

4. 1 and 3

5. All of the above 


\section{Correct! \\ 5. All of the above}

The alcohols are responsible for the majority of the osmolal gap, whereas their organic acid metabolites elevate the anion gap (4). Principles for treatment of poisoning by the alcohols include removal of the alcohol and slow conversion of the alcohols to their toxic acid metabolites by inhibition of alcohol dehydrogenase. Bicarbonate potentially may help to decrease the amount of active organic acid metabolites. Alcohol absorption by the gastrointestinal tract is rapid and gut decontamination is not indicated. However, the alcohols can be removed by hemodialysis.

With the exception of isopropyl alcohol, the alcohols have minimal toxicity in comparison to their metabolites. The alcohols are metabolized first by alcohol dehydrogenase (Figure 2).

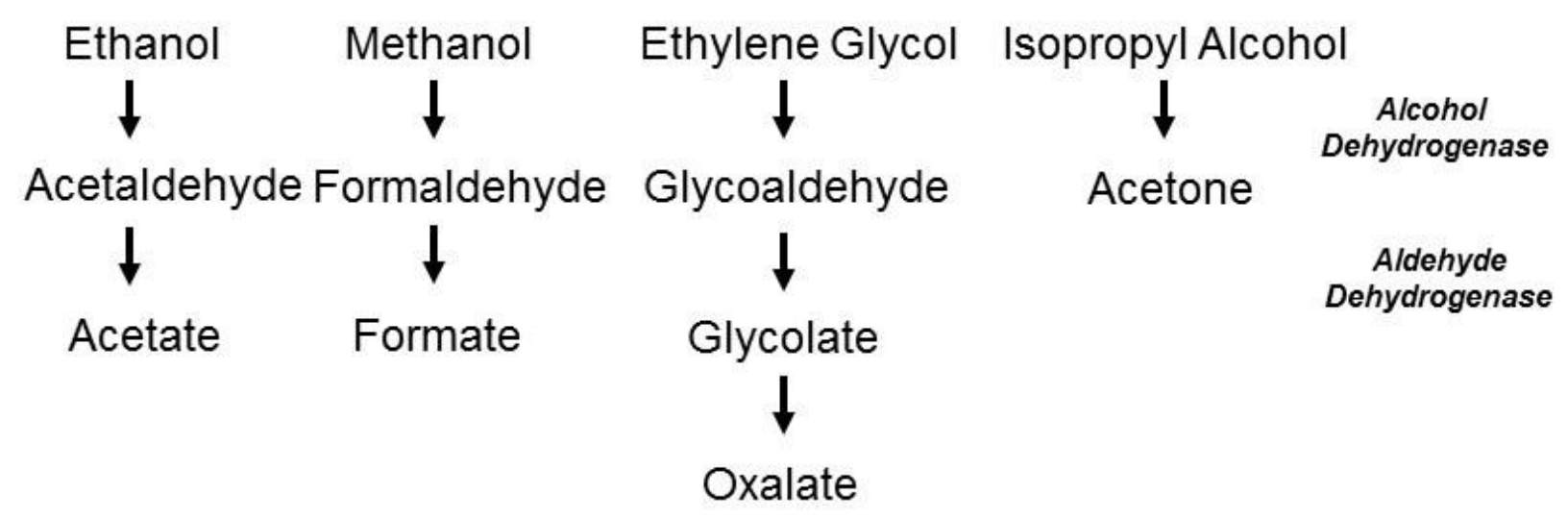

Figure 2. The alcohols are metabolized to aldehydes by alcohol dehydrogenase which are further metabolized by aldehyde dehydrogenase to organic acids.

Inhibition of alcohol dehydrogenase lowers the concentration of the toxic metabolites. Antidote therapy, often using ethanol or fomepizole, is directed towards delaying metabolism until the alcohol is eliminated from the patient's system either naturally or via dialysis.

Our patient was given sodium bicarbonate and begun on fomepizole and hemodialysis. He rapidly improved and was eventually discharged.

\section{References}

1. Emergency Medicine/Altered mental status. Wikibooks. June 15, 2017. Available at: https://en.wikibooks.org/wiki/Emergency Medicine/Altered mental status (accessed 6-27-18).

2. Wilczynski C. Anion gap. Medscape. March 13, 2014. Available at: https://emedicine.medscape.com/article/2087291-overview (accessed 6-27-18). 
3. Osmolal gap calculator. Medscape. Available at: https://reference.medscape.com/calculator/osmolal-gap (accessed 6-27-18).

4. Marts LT, Hsu DJ, Clardy PF. Mind the gap. Ann Am Thorac Soc. 2014 May;11(4):671-4. [CrossRef] [PubMed]

5. Keyes DC. Ethylene glycol toxicity. Medscape. December 05, 2017. Available at: https://emedicine.medscape.com/article/814701-overview (accessed 6-27-18).

6. Korabathina K. Methanol toxicity. Medscape. January 30, 2017. Available at: https://emedicine.medscape.com/article/1174890-overview (accessed 6-27-18). 